\title{
Dephosphorylation of myristoylated alanine-rich $C$ kinase substrate accelerates wound-induced migration of SH-SY5Y cells
}

\author{
Atsuhiro Tanabe ${ }^{1,2^{*}}$, Mitsuya Shiraishi ${ }^{2,3}$, Yasuharu Sasaki ${ }^{2}$ \\ ${ }^{1}$ Division of Biochemistry, Department of Bioscience and Engineering, Shibaura Institute of Technology, Saitama, Japan \\ ${ }^{2}$ Laboratory of Pharmacology, School of Pharmacy, Kitasato University, Tokyo, Japan \\ ${ }^{3}$ Department of Veterinary Pharmacology, Faculty of Agriculture, Kagoshima University, Kagoshima, Japan \\ Email: ${ }^{*}$ tanabea@sic.shibaura-it.ac.jp
}

Received 4 June 2013; revised 4 July 2013; accepted 20 July 2013

Copyright (C) 2013 Atsuhiro Tanabe et al. This is an open access article distributed under the Creative Commons Attribution License, which permits unrestricted use, distribution, and reproduction in any medium, provided the original work is properly cited.

\begin{abstract}
Inflammation, which is induced after infection of bacteria and tissue injury, is one of the important early stages of wound healing. Bradykinin is increased during acute and chronic inflammation. We previously reported that bradykinin stimulation induces dephosphorylation of myristoylated alanine-rich $C$ kinase substrate (MARCKS) after phosphorylation by ROCK leading neurite outgrowth in neuroblastoma SH-SY5Y cells. In this report we showed that knock-down of MARCKS by RNAi reduced cell migration. Wild-type MARCKS-overexpressed SH-SY5Y cells migrated faster than the control cells. Unphosphorylatable MARCKS-overexpressed cells notably migrated fast. Moreover, chronic MARCKS dephosphorylation by a ROCK inhibitor HA-1077 promoted the cell migration, on the other hand a PKC inhibitor Ro-31-8220 did not. After wounding, MARCKS was transiently phosphorylated and dephosphorylated by $20 \mathrm{~min}$. Immunocytochemistry showed that the dephosphorylated MARCKS was localized at neurite tips. These findings suggest that MARCKS dephosphorylation is important in wound-induced migration of SH-SY5Y cells. It indicates the possibility that MARCKS is associated with wound repair after inflammation.
\end{abstract}

Keywords: Wound Healing; MARCKS; Phosphorylation/Dephosphorylation; Cell Migration; Inflammation; ROCK

\section{INTRODUCTION}

Sequence of events in wound healing following tissue

"Corresponding author. damage, which is caused by injury, trauma, tumor or ischemia, includes blood coagulation, inflammation, cell growth, and angiogenesis. Tissue damage releases a mixture of endogenous mediators including bradykinin (BK) and prostaglandin $\mathrm{E}_{2}\left(\mathrm{PGE}_{2}\right)$ into the extracellular space. In wound healing epidermal repair, which is achieved after cell growth and migration, is one of the last events of inflammation [1].

It was demonstrated that BK-induced neurotoxic factors released from astrocytes, including ROS, MMP-9, and $\mathrm{CO}$, may induce neuronal death through a caspase3-dependent apoptotic pathway [2]. On the other hand, the effects of BK on neuron are not perfectly cleared. Recently, we reported that BK-stimulated neuroblastoma SH-SY5Y cells show neurite outgrowth. In the cells myristoylated alanine-rich C kinase substrate (MARCKS) is phosphorylated by ROCK and dephosphorylated by protein phosphatase (PP) 2A [3].

It is well known that PKC, which is activated by phorbolester like TPA and PDBu, phosphorylates MARCKS on Ser159, 163, and 170. Phosphorylated MARCKS detaches from plasma membrane and induces F-actin disruption leading to several cell responses. Reduction of MARCKS with antisense oligonucleotide inhibited phorbolester-induced cell spread [4]. J. C. Gatlin et al. showed that MARCKS inhibited phorbolester-induced growth cone collapse [5]. These data suggest that MARCKS plays a central role in maintenance of cell shape. These effects of MARCKS are phosphorylate status dependent. The importance of MARCKS phosphorylation and phosphorylation mechanisms is well recognized. On the contrary, however, how MARCKS dephosphorylates and the outcome of shift to the unphosphorylated state is not already revealed. We previously showed that MARCKS 
dephosphorylated with insulin-like growth factor (IGF)1 and with BK induced neurite outgrowth in neuroblastoma cell SH-SY5Y [3,6]. Recently it is reported that MARCKS phosphorylation evokes neurotransmitter release [7]. As indicated above, the change of MARCKS phosphorylation level induces cell morphology change leading to cell responses. However, the physical role of MARCKS is still unclear. In this report we showed that MARCKS dephosphorylation induced cell migration in wound healing assay. It indicated the role of MARCKS in inflammation.

\section{MATERIALS AND METHODS}

\subsection{Cell Culture}

SH-SY5Y human neuroblastoma cells (normal-cells), GFP-expressed SH-SY5Y cells (GFP-OE-cells), GFP-fused wild-type MARCKS-overexpressed SH-SY5Y cells (wtMAR-OE-cells), and GFP-fused unphosphorylatable MARCKS-overexpressed cells (m3MAR-OE-cells) [3] were maintained in Dulbecco's modified Eagle's medium supplemented with $10 \%$ fetal bovine serum, 100 units $/ \mathrm{ml}$ penicillin, and $100 \mu \mathrm{g} / \mathrm{ml}$ streptomycin at $37^{\circ} \mathrm{C}$ in a humidified atmosphere with $5 \% \mathrm{CO}_{2}$. When we use protein kinase inhibitors $10 \mu \mathrm{M}$ and $3 \mu \mathrm{M}$ of HA-1077 and Ro31-8220 were added before the scraping, respectively.

\subsection{Wound Healing Assay}

The cells were plated on $35 \mathrm{~mm}$ dish at a density of $8 \times$ $10^{5}$ cells/dish. After $24 \mathrm{~h}, 100 \%$ confluent cells were scraped with pipet tip. After incubation for indicated time the wound area was shot by BZ8000 microscope (Keyence). The width was measured by ImageJ free software.

\subsection{RNA Interference}

MARCKS-specific double-stranded RNA oligonucleotides, each consisting of a 25-nucleotide sense sequence and a 25-nucleotide antisense sequence, were purchased from Invitrogen. The sequences for MARCKS siRNA were as follows: sense, 5'-uucgcugcggucuuggagaacuggg3'; antisense, 5'-cccaguucuccaagaccgcagcgaa-3'.

SH-SY5Y cells were plated on $35 \mathrm{~mm}$ dish at a density of $4 \times 10^{5}$ cells/dish. After $24 \mathrm{~h}, 6.0 \mathrm{pmol}$ of the MARCKS-specific siRNA was transfected to the cells using Lipofectamine 2000 (Invitrogen).

\subsection{Western Blotting Analysis}

Trichloroacetic acid precipitants were subjected to Western blotting analyses as previously described [8]. The phosphorylation of MARCKS was detected with pS159Mar-Ab (1:5000 with Can Get Signal solution 1 (Toyo- bo)). Signals were detected by Chemi Lumi One (Nacalai Tesque) and Light Capture (ATTO). Densitometric analyses were performed using CS Analyzer (ATTO) software.

\section{RESULTS}

\subsection{MARCKS Is Involved in Cell Migration}

At first, we observed the effect of MARCKS knockdown in the wound healing. Treatment by MARCKS siRNA reduced MARCKS protein in neuroblastoma cell line SH-SY5Y cells (Figure 1(a)). The knock-down of MARCKS reduced the cell migration (Figures 1(b) and (c)). Next we observed the migration of SH-SY5Y cells (normal-cell), pEGFP-transfected SH-SY5Y cells (GFPOE-cell), wild-type MARCKS-overexpressed SH-SY5Y cells (wtMAR-OE-cell), and unphosphorylatable MARCKS-overexpressed SH-SY5Y cells (m3MAR-OE-cell) [3] with wound healing assays. The wtMAR- and m30 MAR-OE-cells migrated faster than the normal- and GFP-OE-cells (Figures 2(a) and (b)). These results indicated that MARCKS is involved in wound healing of neuroblastoma cell SH-SY5Y.

\subsection{MARCKS}

\section{Phosphorylation/Dephosphorylation Is Associated with Wound Healing of SH-SY5Y Cells}

MARCKS phosphorylation state was observed after scraping. As shown at Figure 3 MARCKS was phosphorylated slightly $1 \mathrm{~min}$ after scraping and then significantly dephosphorylated by $20 \mathrm{~min}$. The phosphorylation level turned to basal level by $3 \mathrm{~h}$ after scraping (data not shown). Total MARCKS (pan-MARCKS) and $\beta$-actin amount was unaltered.

MARCKS is phosphorylated by both PKC and ROCK in SH-SY5Y cells [8]. In order to observe the effect of MARCKS phosphorylation/dephosphorylation, we used PKC inhibitor Ro-31-8220 and ROCK inhibitor HA1077. HA-1077 treatment increased normal-cells migration besides Ro-31-8220 decreased it (Figures 4(a) and (b)). When we used HA-1077, MARCKS dephosphorylation was continued till $24 \mathrm{~h}$ after scraping, though Ro-31-8220 did not keep MARCKS unphosphorylated (Figure 4(c)). Phase-contrast photomicrographs showed the cells migrated into the wound space with individual directional movement and that HA-1077-treated cells have longer neuritis than untreated and Ro-31-8220treated cells (Figure 4(d)).

\subsection{MARCKS Is Dephosphorylated at Leading Edge}

Finally, we observed MARCKS localization and phos- 


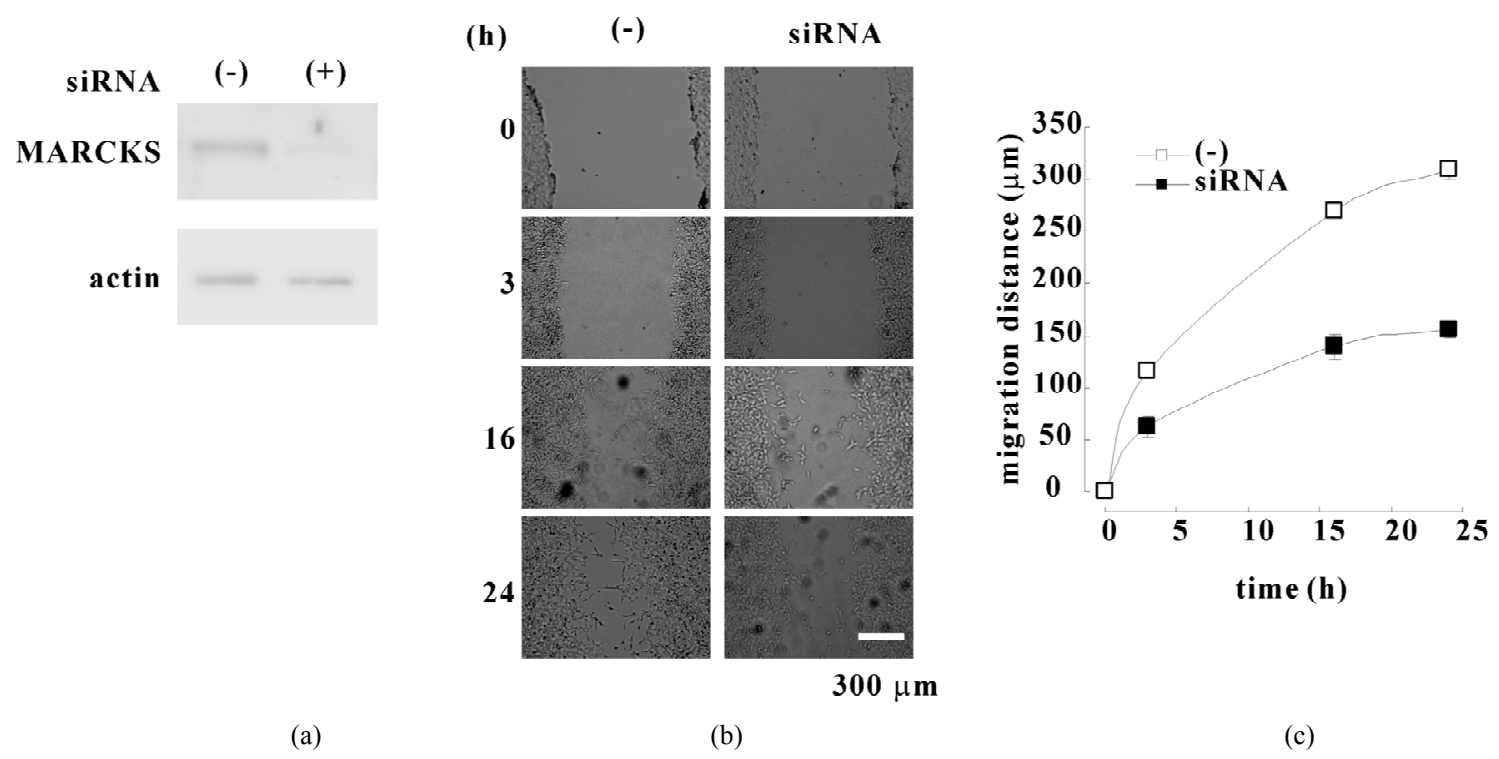

Figure 1. MARCKS knock-down reduces cell migration. (a) MARCKS was knocked-down with MARCKS specific siRNA in SH-SY5Y cells. Twenty-four after transfection MARCKS and $\beta$ actin were detected by Western blotting analyses using MARCKS or $\beta$ actin specific antibody. (b) SH-SY5Y cells and MARCKS-knocked-down cells seeded dishes were scraped. After indicated times the scraped areas were observed. (c) The width of wound in (b) was measured using ImageJ software and migration distances were calculated. The data represent the mean \pm SEM of four independent experiments. ${ }^{*} \mathrm{p}<0.05$ compared with normal-cells.

(h)

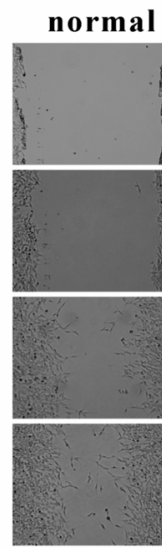

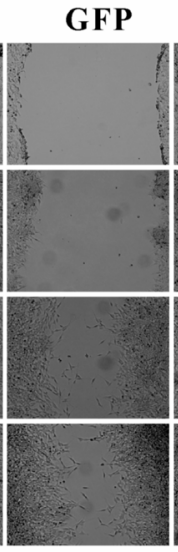
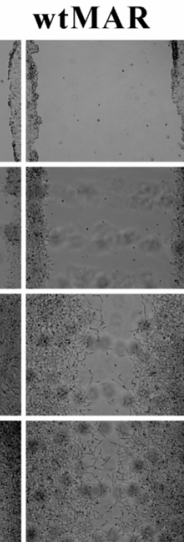

(a)

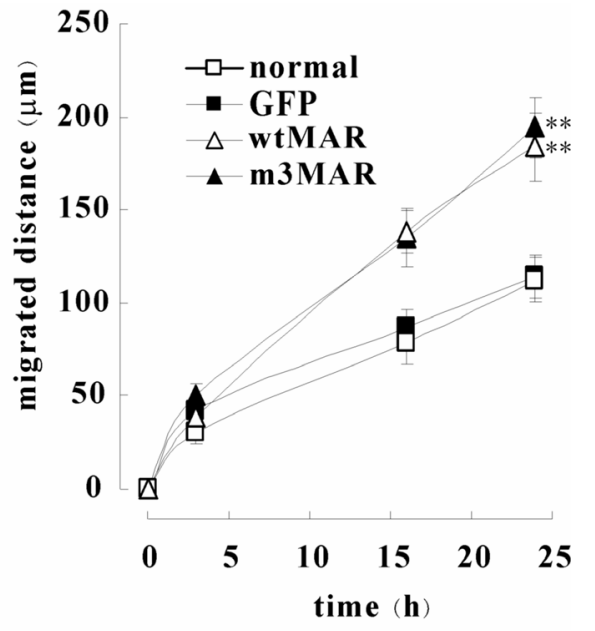

(b)

Figure 2. MARCKS overexpression promotes cell migration. (a) SH-SY5Y cells (normal-cells), GFP-expressed SH-SY5Y cells (GFP-OE-cells), wild-type MARCKS-overexpressed SH-SY5Y cells (wtMAR-OE-cells), and unphosphorylatable MARCKS-overexpressed cells (m3MAR-OE-cells) seeded dishes were scraped. After indicated times the scraped areas were observed. (b) The width of wound in (a) was measured using ImageJ software and migration distances were calculated. The data represent the mean \pm SEM of four independent experiments. ${ }^{*} p<0.01$ compared with normal-cells.

phorylation state in the cells by immunocytochemistry (Figure 5). Just after scraping (0 min) MARCKS existed at plasma membrane (pan-MARCKS; green) and it was phosphorylated (p-MARCKS; red). At 5 min though MARCKS existed plasma membrane, phosphorylated MARCKS was defused to cytosol. Twenty min after scraping lamellipodia like morphology was appeared and
MARCKS was accumulated at the edge. The MARCKS at lamellipodia was not phosphorylated (Figure $\mathbf{5}$ arrow heads). Three hours after scraping the cells extended neurite toward wound space. At neurite tip there was MARCKS and the MARCKS was dephosphorylated. The phosphorylated MARCKS mainly existed at the cell bodies. 


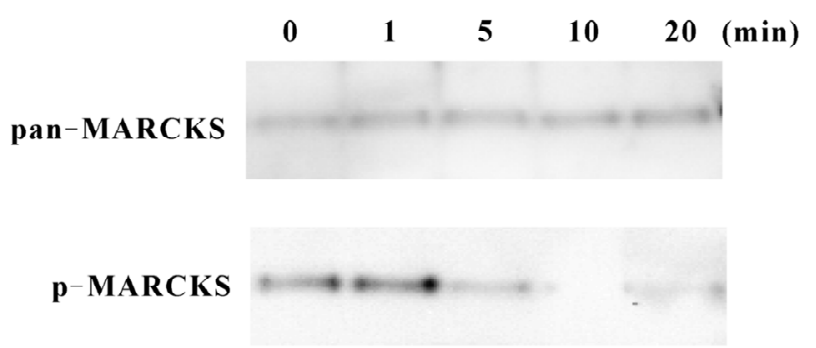

Bactir

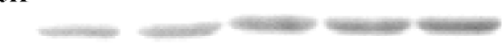

(a)

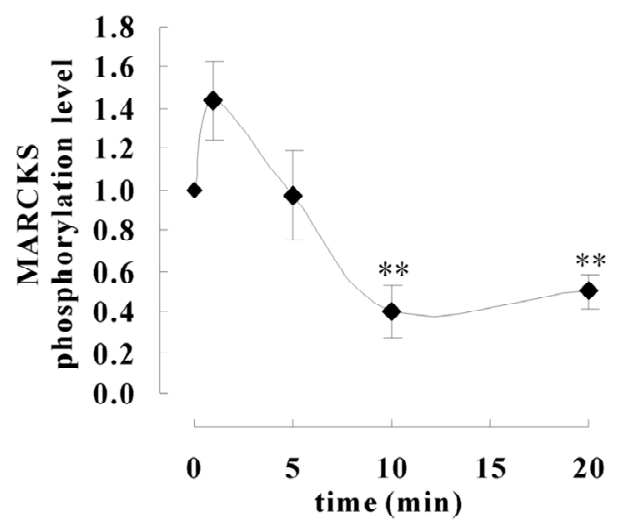

(b)

Figure 3. MARCKS is dephosphorylate during wound healing. (a) SH-SY5Y cell seeded dishes were scraped with pippet tips after indicated times the cell extracts were applied to Western blot analyses using anti-MARCKS antibody, pS159-Mar-Ab, and anti- $\beta$ actin antibody. (b) Signals in (a) were analysed using CS Analyzer (ATTO) software. The data represent the mean \pm SEM of three independent experiments. ${ }^{* *} p<0.01$ compared with $0 \mathrm{~min}$.

\section{DISCUSSION}

Myristoylated alanine-rich C kinase substrate (MARCKS) is thought to regulate cellular adhesion, spreading, proliferation, and migration through its interaction to cytoskeleton. MARCKS mediates epidermal growth factor receptor variant III-induced glioma cell invasion [9] and vascular smooth muscle migration [10]. Conversely, MARCKS had no effect on endothelial cells migration [10]. As these reports indicate, it is not unclear whether MARCKS promotes cell migration or inhibits it and how MARCKS regulates cell migration. In this study we investigated the effect of MARCKS phosphorylation state on neuroblastoma SH-SY5Y cells migration in wound healing assay.

In this experience wild-type MARCKS-overexpressed cells (wtMAR-OE-cell) and unphosphorylatable MARCKS-overexpressed cells (m3MAR-OE-cell) migrated faster than normal SH-SY5Y cells (normal-cell) and vector transfected cells (GFP-OE-cell) (Figure 2). These results suggest that dephosphorylated MARCKS is involved in neuroblastoma migration as we previously reported that dephosphorylated MARCKS is involved in Insulin like growth factor-I induced neurite outgrowth which is the first step of cell migration [6]. As shown at Figure 3, MARCKS was dephosphorylated transiently after scraping. At the beginning of neurite outgrowth and migration the cells showed lamellar protrusions. Twenty minutes after scraping SH-SY5Y cells showed lamellar protrusions and MARCKS were accumulated on them (Figure 4 arrows). The MARCKS phosphorylation level on lamellar protrusions is low (Figure $\mathbf{4}$ arrow heads). However, the localization of the phosphorylated MARCKS was different from that at just after scraping. Just after scraping phosphorylated MARCKS existed plasma

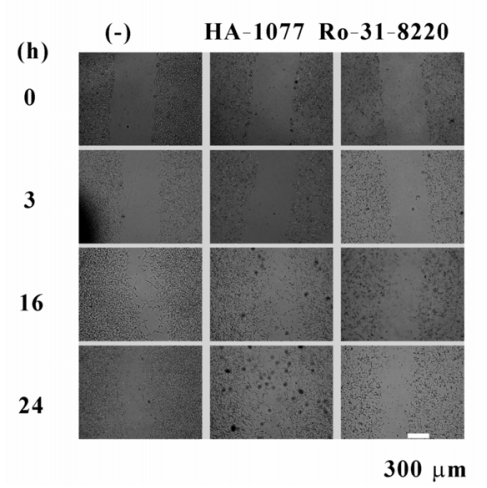

(a)

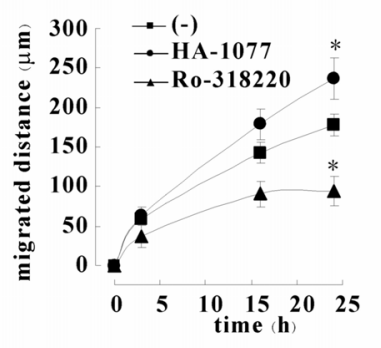

(b)

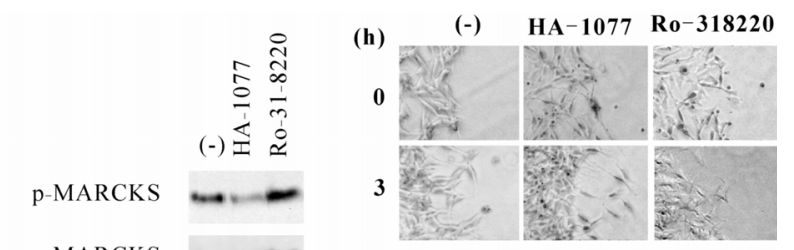

pan-MARCKS

Bactin

(c) (d)

Figure 4. Chronicle MARCKS dephosphorylation promotes cell migration and neurite outgrowth. (a) SH-SY5Y cells were pretreated with $10 \mu \mathrm{M}$ HA-1077 or $3 \mu \mathrm{M}$ Ro-31-8220 before scraping. After indicated times the scraped areas were observed. (b) The width of wound in (a) was measured using ImageJ software and migration distances were calculated. The data represent the mean \pm SEM of four independent experiments. ${ }^{*} \mathrm{p}<0.05$ compared with untreated cells. (c) SH-SY5Y cells were treated with $10 \mu \mathrm{M}$ HA-1077 or 3 $\mu \mathrm{M}$ Ro-31-8220 for 24 hours. The cell extracts were applied to Western blot analyses using anti-MARCKS antibody, pS159-Mar-Ab, and $\beta$ actin antibody. 

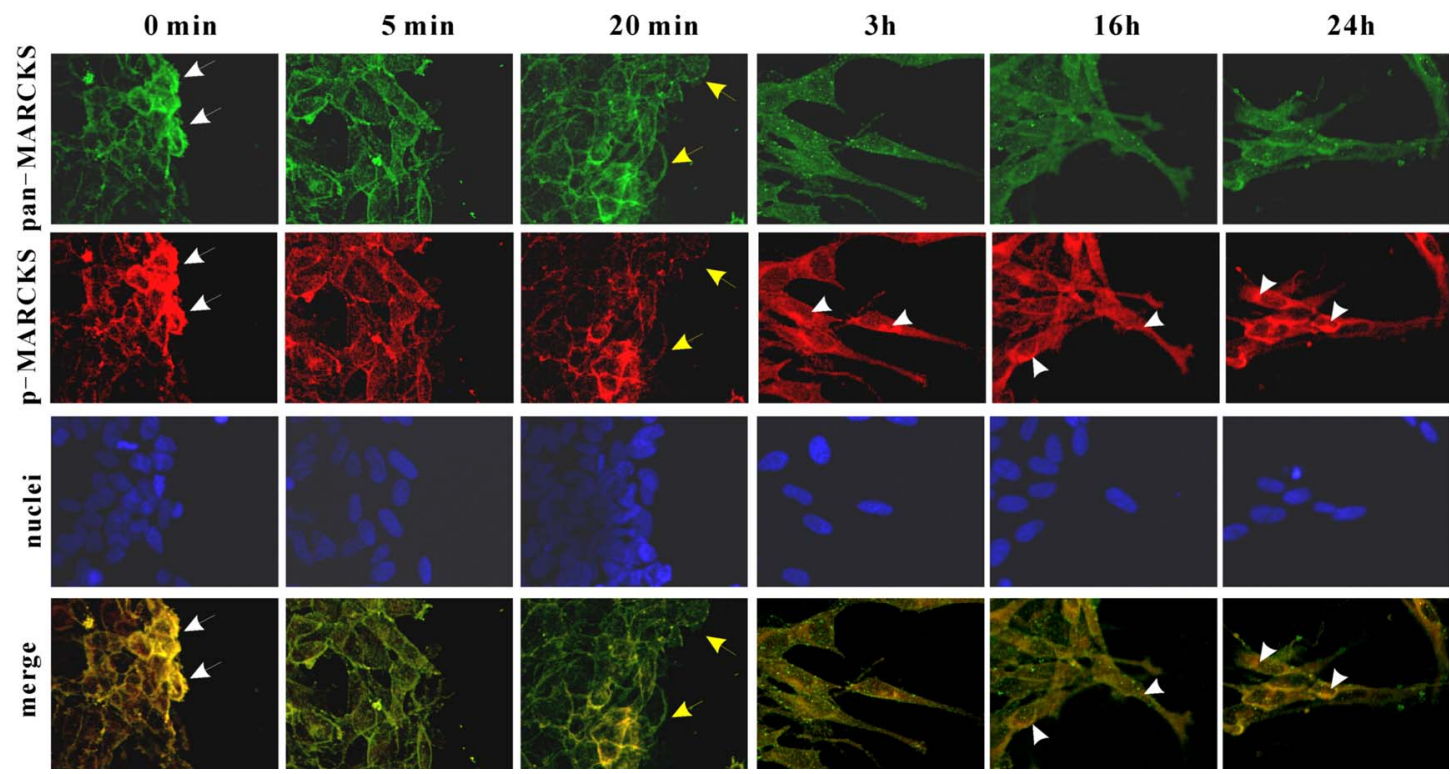

Figure 5. MARCSK is dephosphorylated in leading edge. After cell scraping MARCKS, phosphorylated MARCKS and nuclei were stained with anti-MARCKS antibody and FITC-conjugated anti-goat IgG antibody (green), pS159-Mar-Ab and anti-mouse IgG antibody, and DAPI, respectively.

membrane though it is well known that phosphorylated MARCKS moves to cytosol, whereas at $3 \mathrm{~h}$ after scraping the cells extended the cell body to the scratched area and then phosphorylated MARCKS localized at cytosol (Figure 5). Then, in the neurite MARCKS was detected, however, MARCKS in the neurite was not phosphorylated (Figure 5 yellow arrows). It supported our previous data that unphosphorylated MARCKS is involved in neurite outgrowth [6].

We have reported that MARCKS is phosphorylated by both PKC and ROCK in SH-SY5Y cells [8]. We performed wound healing assay under the condition when ROCK inhibitor HA-1077 or PKC inhibitor Ro-31-8220 existed because we previously observed that both $10 \mu \mathrm{M}$ HA-1077 and $3 \mu \mathrm{M}$ Ro-31-8220 decreased basal MARCKS phosphorylation level [8], in this study we used them at that concentration. HA-1077 induced the migration rate conversely and Ro-31-8820 decreased it (Figures 4(a) and (b)). However, $24 \mathrm{~h}$ after scraping, only HA1077 sustained MARCKS in low phosphorylated state (Figure 4(c)). Additionally, HA-1077-treated cells showed longer neurites comparing with those of untreated cells at $24 \mathrm{~h}$ after scraping. Oppositely, the neurites of Ro-31-8220-treated cells were shorter (Figure 4(d)). These results conformed to the notion that unphosphorylated MARCKS induces neurite outgrowth and cell migration. It has been believed that ROCK, which is the downstream of small G-protein RhoA, is involved in cell membrane blebing and actin stress fiber. Recently, it has been reported that ROCK mediates cell migration $[11,12]$. The indicated substrates of ROCK in cell migration are paxillin and Pyk-2. In this study we suggest that
MARCKS is another candidate of ROCK substrate in cell migration [12].

Inflammatory mediators, like $\mathrm{BK}, \mathrm{PGE}_{2}$ or serotonin (5-HT) generate pain [13]. Treatment of SH-SY5Y cells with BK induces neurite outgrowth via transient MARCKS phosphorylation [3]. $\mathrm{PGE}_{2}$ treatment also induces MARCKS phosphorylation in SH-SY5Y cells (unpublished data). Therefore, we consider that MARCKS is associated with inflammation and/or pain. The relationship between MARCKS and inflammation has been reported recently $[7,14,15]$. These reports and our results suggest that response against inflammation including wound healing is one of the physical roles of MARCKS.

\section{ACKNOWLEDGEMENTS}

We thanks Dr. Naoaki Saito for wtMARCKS and m3MARCKS express plasmid. This work was supported in part by Shibaura Institute of Technology Project Research Grant (start-up).

\section{REFERENCES}

[1] Paul, M. (1997) Wound healing-Aiming for perfect skin regeneration. Science, 276, 75-81. doi:10.1126/science.276.5309.75

[2] Chuen-Mao, Y., Hsi-Lung, H., Chih-Chung, L., RueyHorng, S., Pei-Ling, C., Shin-Ei, C. and Li-Der, H. (2013) Multiple factors from bradykinin-challenged astrocytes contribute to the neuronal apoptosis: Involvement of astroglial ROS, MMP-9 and HO-1/CO system. Molecular Neurobiology, 47, 1020. doi:10.1007/s12035-013-8402-1

[3] Tanabe, A., Shiraishi, M., Negishi, M., Saito, N., Tanabe, 
M. and Sasaki, Y. (2012) MARCKS dephosphorylation is involved in bradykinin-induced neurite outgrowth in neuroblastoma SH-SY5Y cells. Journal of Cellular Physiology, 227, 618-629. doi:10.1002/jep.22763

[4] Disatnik, M., Boutet, S.C. and Rando, T.A. (2002) Alpha 5 beta 1 integrin mediates muscle cell spreading through PKC and MARCKS signaling pathway. Molecular Biology of the Cell, 13, 349A-349A.

[5] Gatlin, J.C., Estrada-Bernal, A., Sanford, S.D. and Pfenninger, K.H. (2006) Myristoylated, alanine-rich C-kinase substrate phosphorylation regulates growth cone adhesion and pathfinding. Molecular Biology of the Cell, 17, 51155130. doi:10.1091/mbc.E05-12-1183

[6] Shiraishi, M., Tanabe, A., Saito, N. and Sasaki, Y. (2006) Unphosphorylated MARCKS is involved in neurite initiation induced by insulin-like growth factor-I in SHSY5Y cells. Journal of Cellular Physiology, 209, 10291038. doi:10.1002/jep.20814

[7] Green, T.D., Crews, A.L., Park, J., Fang, S.J. and Adler, K.B. (2011) Regulation of mucin secretion and inflammation in asthma: A role for MARCKS protein? Biochimica ET Biophysica Acta-General Subjects, 1810, 11101113. doi:10.1016/j.bbagen.2011.01.009

[8] Tanabe, A., Kamisuki, Y., Hidaka, H., Suzuki, M., Negishi, M. and Takuwa, Y. (2006) PKC phosphorylates MARCKS Ser159 not only directly but also through RhoA/ROCK. Biochemical and Biophysical Research Communications, 345, 156-161. doi:10.1016/i.bbrc.2006.04.082

[9] Micallef, J., Taccone, M., Mukherjee, J., Croul, S., Busby, J., Moran, M.F. and Guha, A. (2009) Epidermal growth factor receptor variant III-induced glioma invasion is mediated through myristoylated alanine-rich protein kinase C substrate overexpression. Cancer research, 69, 75487556. doi:10.1158/0008-5472.CAN-08-4783

[10] Monahan, T.S. andersen, N.D., Martin, M.C., Malek, J.Y.,
Shrikhande, G.V., Pradhan, L., Ferran, C. and LoGerfo, F.W. (2009) MARCKS silencing differentially affects human vascular smooth muscle and endothelial cell phenotypes to inhibit neointimal hyperplasia in saphenous vein. FASEB Journal: Official Publication of the Federation of American Societies for Experimental Biology, 23, 557-564.

[11] Hayashi, M., Okabe, K., Kato, K., Okumura, M., Fukui, R., Fukushima, N. and Tsujiuchi, T. (2012) Differential function of lysophosphatidic acid receptors in cell proliferation and migration of neuroblastoma cells. Cancer Letters, 316, 91-96. doi:10.1016/j.canlet.2011.10.030

[12] Rebecca, A.W. and Keith, B. (2003) RhoA and ROCK promote migration by limiting membrane protrusions. Journal of Biological Chemistry, 278, 13578-13584. doi:10.1074/jbc.M211584200

[13] Mizumura, K. and Kumazawa, T. (1996) Modification of nociceptor responses by inflammatory mediators and second messengers implicated in their action-A study in canine testicular polymodal receptors. Elsevier Science Bv, Amsterdam, 115-141.

[14] Techasen, A., Loilome, W., Namwat, N., Duenngai, K., Cha'on, U., Thanan, R., Sithithaworn, P., Miwa, M. and Yongvanit, P. (2012) Opisthorchis viverrini-antigen induces expression of MARCKS during inflammation-associated cholangiocarcinogenesis. Parasitology International, 61, 140-144. doi:10.1016/j.parint.2011.07.004

[15] Damera, G., Panettieri, R.A., Jester, W.F., Jiang, M.Q., Zhao, H.J., Fogle, H.W., Mittelman, M., Haczku, A., Murphy, E. and Parikh, I. (2010) Inhibition of myristoylated alanine-rich $\mathrm{C}$ kinase substrate (MARCKS) protein inhibits ozone-induced airway neutrophilia and inflammation. Experimental Lung Research, 36, 75-84. doi:10.3109/01902140903131200 